\title{
Blockchain Reality, Its Strategic Value and Busting the Myth Around the White Elephant
}

\author{
Virbahu Nandishwar Jain \\ CPIM MIE CE \\ Hanover, NH, USA
}

\begin{abstract}
Blockchain is disrupting the normal day to day operations for every organization which is adopting to its latest trend. Each company is different and evolving in their own predominantly defined spaces. The real question arises, how this change will preclude the Blockchain bubble to burst or is there any bubble, yet? Honestly, Blockchain Disruption is an overused term in today's world and disruption fatigue is real. But disruption is also incredibly relevant and important, what do CXO's need to do during this time is not to ignore it. Get clarifications about their doubts on the expectations vs. reality, so that they can be the change management agents to steer the discussion how it matters and how it is going to impact the day to day business operations? The real question is what is that we are disrupting, what is changing and who should pay attention and why?
\end{abstract}

Keywords- Blockchain, C-Suite, Strategic Planning, Disruption Technologies, Change Management

\section{INTRODUCTION}

Should I or Should I not? What do we gain from it, should we be looking at short term benefits or long terms gains? How reliable is Blockchain? Is it stable? Are we the piggybacks for testing the Blockchain technology? These are some of the questions which goes on to the mind of every C-Level Industrial prospects and rightly so, when it comes to adopting the new and upcoming technologies. Managing Technology as a Business Strategy is the way forward in the $21^{\text {st }}$ Century. It assures its purpose with the overall alignment of the business goals and Shareholder Value. The management of technology is analogous to the management of investment. The development and use of technology must be guided explicitly by the business strategy of the firm; at the same time, technological developments should help define the opportunities and threats to which the strategy should then respond. Thus, the strategic management of technology involves a dialogue - a process through which both the strategic targets of the enterprise and the goals of its technology program are regularly reviewed and revised. The reality is, disruption is always good for someone. But it is not inherently good or bad. For some it's an opportunity, for others it's a threat. Don't dwell on the negative. Instead, take advantage of the possibilities the disruption creates.

\section{A. Early Adopters and Blockchain Evolution}

Only 1 percent of CIOs indicated any kind of blockchain adoption within their organizations, and only 8 percent of CIOs were in short-term planning or active experimentation with blockchain, according to Gartner's 2018 CIO Survey. Furthermore, 77 percent of CIOs surveyed said their organization has no interest in the technology and/or no action planned to investigate or develop it. [2]

Among 293 CIOs of organizations that are in short-term planning or have already invested in blockchain initiatives, 23 percent of CIOs said that blockchain requires the newest skills to implement of any technology area, while 18 percent said that blockchain skills are the most difficult to find. A further 14 percent indicated that blockchain requires the greatest change in the culture of the IT department, and 13 percent believed that the structure of the IT department had to change in order to implement blockchain. [2]

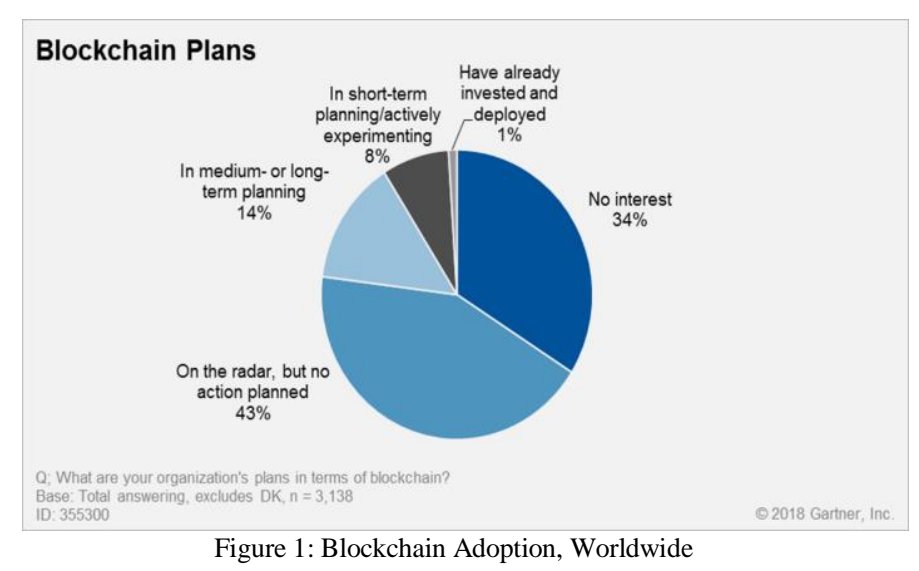

To understand the blockchain evolution trajectory, it's helpful to review the Hype Cycle for Blockchain Business, 2018. For all the hype and possibility of blockchain, the technology barely registers as a priority for CIOs. From, Gartner 2019 CIO survey, only 5\% of CIOs rated blockchain as a game changer for their organizations, far below artificial intelligence, cloud, and data and analytics. Across all categories of $\mathrm{CIO}$ respondents (top, typical and trailing performers), $11 \%$ have deployed or will deploy blockchain in the next twelve months.

To help CIOs understand today's blockchain reality and the future evolution, please see the Gartner blockchain spectrum which discusses how the blockchain will evolve until 2030

\section{B. The Future and Expectations}

\section{1) The Early Phases : Business Enablers, 2009-2020}

a) This early phase of blockchain-enabled experiments are built on top of existing systems to reduce cost and friction in private, proprietary activities. They have only limited distribution capabilities to a small number of nodes either within or between enterprises. [4] 
2) The Current Phase : Decentralization, 2016-2023

The current phase of blockchain-inspired solutions are usually designed to address a specific operational issue most often in terms of inter-organizational process or record keeping inefficiency. These solutions have tokenization or decentralized decision making. "They don't want to give up centralized control".[4]

\section{3) The Future Stabilization : 2020 and Beyond}

a) Blockchain Complete offerings, starting in the 2020s, will implement smart contracts and deliver the full value proposition of blockchain including decentralization and tokenization. [4]

\section{4) The Autonomous Operations : Post 2025}

a) In this future state, smart contracts will have real autonomy and advanced technologies will enable exchanges and transactions that aren't currently possible. This is when we'll start to see Decentralized Autonomous Organizations (DAO) and microtransactions performed by machines. [4]

\section{5) Beyond : Post Stabilization}

a) It is expected that blockchain will be the driving force in changing the way, businesses are operated in current worls, but we are far away from reality. Once, in its true implementation form, this will deliver fully trusted netwrok in a fast moving economies.

\section{Hype Cycle for Blockchain Business, 2018}

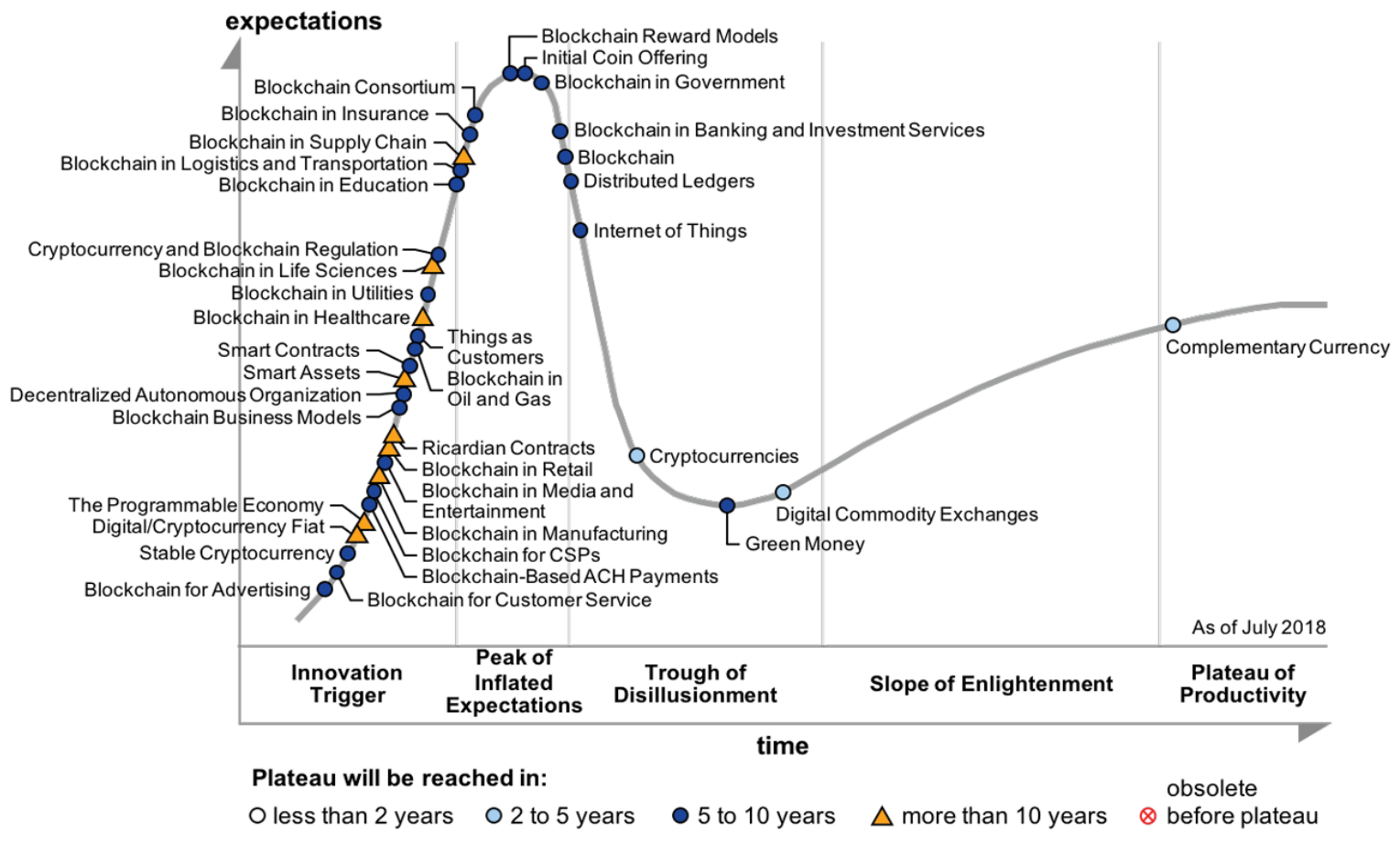

\section{gartner.com/SmarterWithGartner}

C 2018 Gartner, Inc. and/or its affiliates. All rights reserved. Gartner is a registered trademark of Gartner, Inc. or its affiliates.

\section{Gartner}

Figure 2: Blockchain trajectory and the Future it holds for the Hype Cycle for Blockchain Business

C. The Challenges and Myths around Blockchain

The digital disruption has started making waves and the most important thing today is to face it, CXO's of the new age cannot turn a blind eye to it, while ostensibly a long-term gain and a definite reward in the future, the executives needs to be prepared for this white elephant, from Change management and business process re-engineering prospective.

By and large, this is one of the biggest ever disruption causing IT innovation that has happened to the $21^{\text {st }}$ century. 
With the "ICO", the technology has gone around door to door like a Loose Cannon, but now, the Organizations are getting more matured and understanding the use and benefits of blockchain in many other areas for the betterment of humankind. Blockchain has several use cases which was described in the first series paper by "Virbahu Nandishwar Jain". [1]

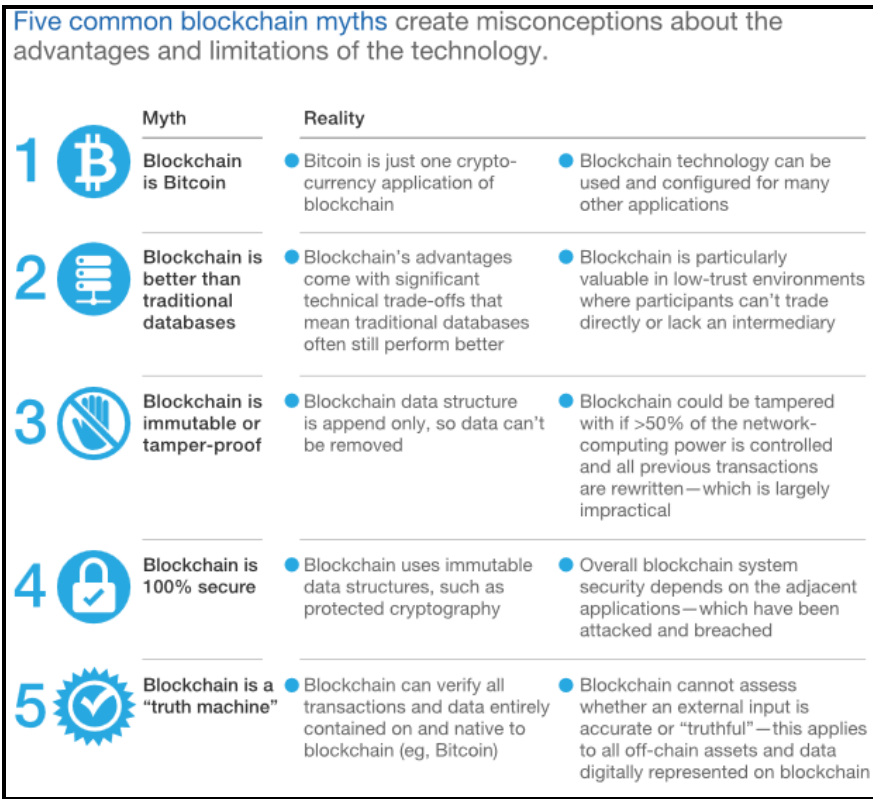

Figure 3: Mckinsey\&Company, Blockchain beyond the Hype

"Disruption is a bad thing". The reality is, disruption is always good for someone. But it is not inherently good or bad. For some it's an opportunity, for others it's a threat. Don't dwell on the negative. Instead, take advantage of the possibilities the disruption creates. [3]

"Disruption is an overused buzzword". Disruption is an overused term and disruption fatigue is real. But disruption is also incredibly relevant and important. Don't ignore it. Ask questions to steer the discussion to what matters. What is being disrupted and how important is the disruption? What's the fundamental shift? Who should pay attention and why? [3]

"Any change is a disruption". Change does not equal disruption. Disruption occurs when there is a fundamental shift in a system or environment, for example cloud computing or smartphones. Often what is perceived as disruption is actually a feature or a fad such as 3D T.V. or Pokémon Go. Ensure your strategy reflects the post-disruption landscape. [3]

"Disruption is a technology only issue". While digital disruption will almost always have some element of technology, the technology itself is not necessarily the primary disruptive element. View disruptions in the context of what models have been disrupted in four elements of disruption: business, technology, industry and society. [3]

"Disruption is only for the digital giants". Digital giants, such Amazon, Google and Apple, might disrupt first, but the bulk of disruption happens across thousands of small medium and large companies. Pay attention to and learn from these digital giants. Create action plans that lead to organizational, cultural, financial or business model change to begin the process of exploiting a disruption. Examine also the secondary effects of disruptions, which affect a broad range of companies and individuals. [3]

"Digital disruption happens only in consumer markets". The reality is digital disruption is frequently first seen in consumer markets, but its impact extends into many different markets and businesses. This is largely a result of the phenomenon known as the consumerization of IT, or when consumer disruptions are adopted by IT. Put yourself in the users' shoes and apply consumer scenarios to understand digital disruption in B2B. [3]

"The most hyped disruptions are the most disruptive". Artificial intelligence, blockchain and virtual reality receive a lot of hype and visibility. But they are not mature enough to be considered true disruptions - yet. True disruptions have mainstream adoption as well secondary effects. While hype declines over time, those secondary effects do not. [3]

"Innovation, transformation and disruption are all the same thing". Innovation and transformation are two different things that exist in a virtuous cycle. Sometimes one interrupts or guides the other, resulting in disruption. Don't lose sight of the link between the two. Incorporate disruption analysis into your innovation and/or transformation efforts. [3]

"Disruption is someone else's problem". Disruption affects everyone. CIOs and IT leaders are often uniquely qualified to identify it. However, it's not always the CIO or IT staff that encounters disruptors or disruptive opportunities as they occur in areas other than technology. Work with enterprise architects and chief strategy officers to forge a strategy for leveraging disruptions and establish yourself as a disruptor CIO. [3]

"We can't be disrupted". Anyone can be disrupted, even disruptors. No one is unassailable. Apple must contend with defensive, disruptive tactics from Google, whose Android competes with the iPhone. Visionaries recognize that they can be disrupted and employ self-disrupting strategies to ensure they benefit from the next fundamental change. Avoid arrogance and do not be afraid to self-disrupt. [3]

\section{FUTURE OUTLOOK AND STRATEGIC VALUE OF BLOCKCHAIN}

This section gives an outlook into the near future of blockchain technology by discussing the information and partly differing opinions received from several leading researchers and industry experts.

. Some of the Global Regions, who are getting into the Blockchain world and the Investment which has been put forth into developing Blockchain technologies by region and Industry will give us a glimpse of the focus the world is moving into the disruption due to Blockchain technologies.

"Despite the hype, blockchain is still an immature technology, with a market that is still nascent and a clear recipe for success that has not yet emerged. Unstructured experimentation of blockchain solutions without strategic evaluation of the value at stake or the feasibility of capturing it means that many companies will not see a return on their investments. How can companies determine if there is strategic value in blockchain that justifies major investments?

Based on Mckinsey\&Company research to seek answer for not only the strategic importance of blockchain to major 
industries but also who can capture what type of value through what type of approach. In-depth, industry-by-industry analysis combined with expert and company interviews revealed more than 90 discrete use cases of varying maturity for blockchain across major industries.

The below analysis suggests the following three key insights on the strategic value of blockchain:

1. Blockchain does not have to be a disintermediated to generate value, a fact that encourages permissioned commercial applications.

2. Blockchain's short-term value will be predominantly in reducing cost before creating transformative business models.

3. Blockchain is still three to five years away from feasibility at scale, primarily because of the difficulty of resolving the "coopetition" paradox to establish common standards.
Companies should take the following structured approach in their blockchain strategies:

1. Identify value by pragmatically and skeptically assessing impact and feasibility at a granular level and focusing on addressing true pain points with specific use cases within select industries.

2. Capture value by tailoring strategic approaches to blockchain to their market position, with consideration of measures such as ability to shape the ecosystem, establish standards, and address regulatory barriers.

3. With the right strategic approach, companies can start extracting value in the short term. Dominant players who can establish their blockchains as the market solutions should make big bets now.

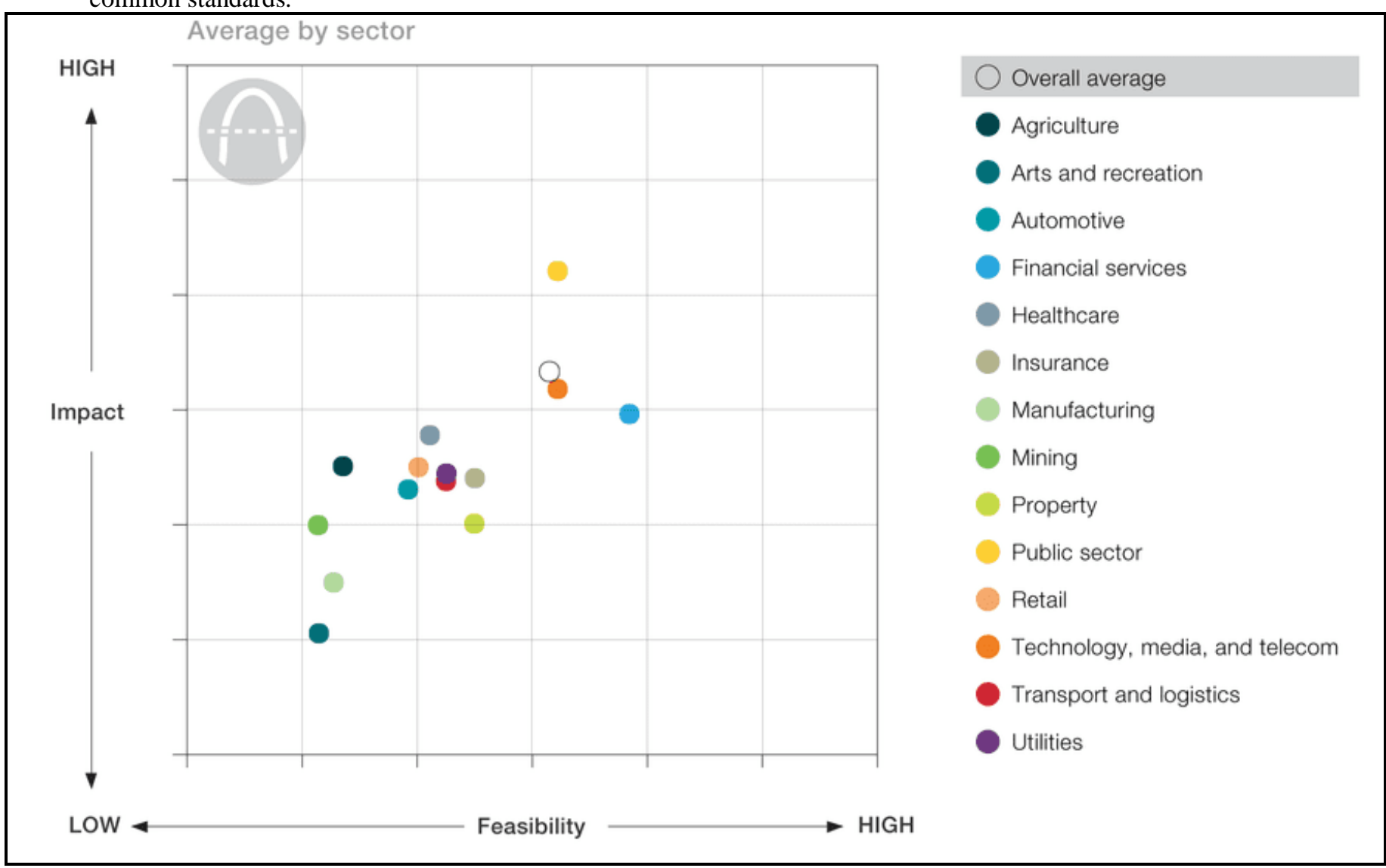

Figure 4: Fintech, Mckinsey\&Company, Blockchain beyond the hype: What is the strategic business value?

A. How to compete: Optimize blockchain strategy based on market position

- Once companies have identified promising use cases, they must develop their strategies based on consideration of their market positions relative to their target use cases. Many of the feasibility factors already discussed are within a business's sphere of influence; even technology and asset constraints can be managed through trade-offs and a series of design choices to shape a viable solution.

- Therefore, a company's optimal strategic approach to blockchain will fundamentally be defined by the following two market factors, which are those they can least affect:

- market dominance - the ability of a player to influence the key parties of a use case

- standardization and regulatory barriersthe requirement for regulatory approvals or coordination on standards

- These two factors are critical in determining a company's optimal strategic approach because they are integral to achieving the coordination required. Blockchain's value comes from its network effects and interoperability, and all parties need to agree on a common standard to realize this value-multiple siloed 
blockchains provide little advantage over multiple siloed databases. As the technology develops, a market standard will emerge, and investments into the nondominant standard will be wasted.

\begin{tabular}{|l|l|l|}
\hline Optimal blockchain strategy for each use case is dependent on market \\
position and ability to influence standards and regulatory barriers.
\end{tabular}

Figure 5: Fintech, Mckinsey\&Company, Optimization

\section{B. Blockchain does not need to be a disintermediator to} generate value

- Benefits from reductions in transaction complexity and cost, as well as improvements in transparency and fraud controls can be captured by existing institutions and multiparty transactions using appropriate blockchain architecture. The economic incentives to capture value opportunities are driving incumbents to harness blockchain rather than be overtaken by it. Therefore, the commercial model that is most likely to succeed in the short term is permissioned rather than public blockchain. Public blockchains, like Bitcoin, have no central authority and are regarded as enablers of total disruptive disintermediation. Permissioned blockchains are hosted on private computing networks, with controlled access and editing rights.

\begin{tabular}{|c|c|c|c|}
\hline \multirow{2}{*}{\multicolumn{2}{|c|}{$\begin{array}{l}\text { Most commercial } \\
\text { to optimize netwo } \\
\begin{array}{l}\text { Blockchain- } \\
\text { architecture options }\end{array}\end{array}$}} & $\begin{array}{l}\text { cchain will use private, } \\
\text { enness and scalability. }\end{array}$ & issioned architecture \\
\hline & & \multicolumn{2}{|c|}{$\begin{array}{l}\text { Architecure based on read, write, or commit } \\
\text { permissions granted to the participants }\end{array}$} \\
\hline \multirow{3}{*}{$\begin{array}{l}\text { Architecture } \\
\text { based on } \\
\text { ownership } \\
\text { of the data } \\
\text { infrastructure }\end{array}$} & & Permissionless & Permissioned \\
\hline & Public & $\begin{array}{l}\text { - Anyone can join, read, write, } \\
\text { and commit } \\
\text { - Hosted on public servers } \\
\text { - Anonymous, highly resilient } \\
\text { Low scalability }\end{array}$ & $\begin{array}{l}\text { Anyone can join and read } \\
\text { Only authorized and } \\
\text { known participants can } \\
\text { write and commit } \\
\text { Medium scalability }\end{array}$ \\
\hline & Private & $\begin{array}{l}\text { Only authorized } \\
\text { participants can join, } \\
\text { read, and write } \\
\text { Hosted on private servers } \\
\text { High scalability }\end{array}$ & $\begin{array}{l}\text { Only authorized partici- } \\
\text { pants can join and read } \\
\text { Only the network operator } \\
\text { can write and commit } \\
\text { Very high scalability }\end{array}$ \\
\hline
\end{tabular}

Figure 6: Fintech, Mckinsey\&Company, Architecture

\section{In the short term, blockchain's strategic value is} mainly in cost reduction

- Blockchain might have the disruptive potential to be the basis of new operating models, but its initial impact will be to drive operational efficiencies. Cost can be taken out of existing processes by removing intermediaries or the administrative effort of record keeping and transaction reconciliation. This can shift the flow of value by capturing lost revenues and creating new revenues for blockchain-service providers. Based on our quantification of the monetary impact of the more than 90 use cases we analyzed, we estimate approximately 70 percent of the value at stake in the short term is in cost reduction, followed by revenue generation and capital relief.

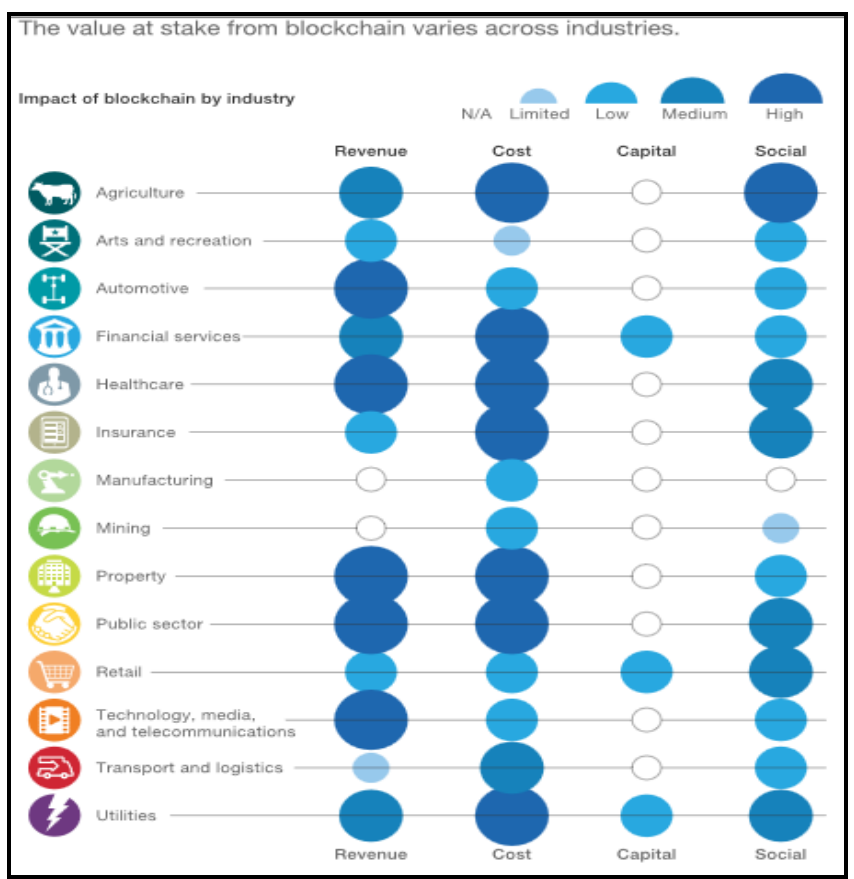

Figure 7: Fintech, Mckinsey\&Company, Value at Stake

D. Feasibility at scale is likely to be three to five years away

- The strategic value of blockchain will only be realized if commercially viable solutions can be deployed at scale. Our analysis evaluated each of the more than 90 potential use cases against the four key factors that determine a use case's feasibility in each industry: standards and regulations, technology, asset, and ecosystem. While many companies are already experimenting, meaningful scale remains three to five years away for several key reasons." [5] 


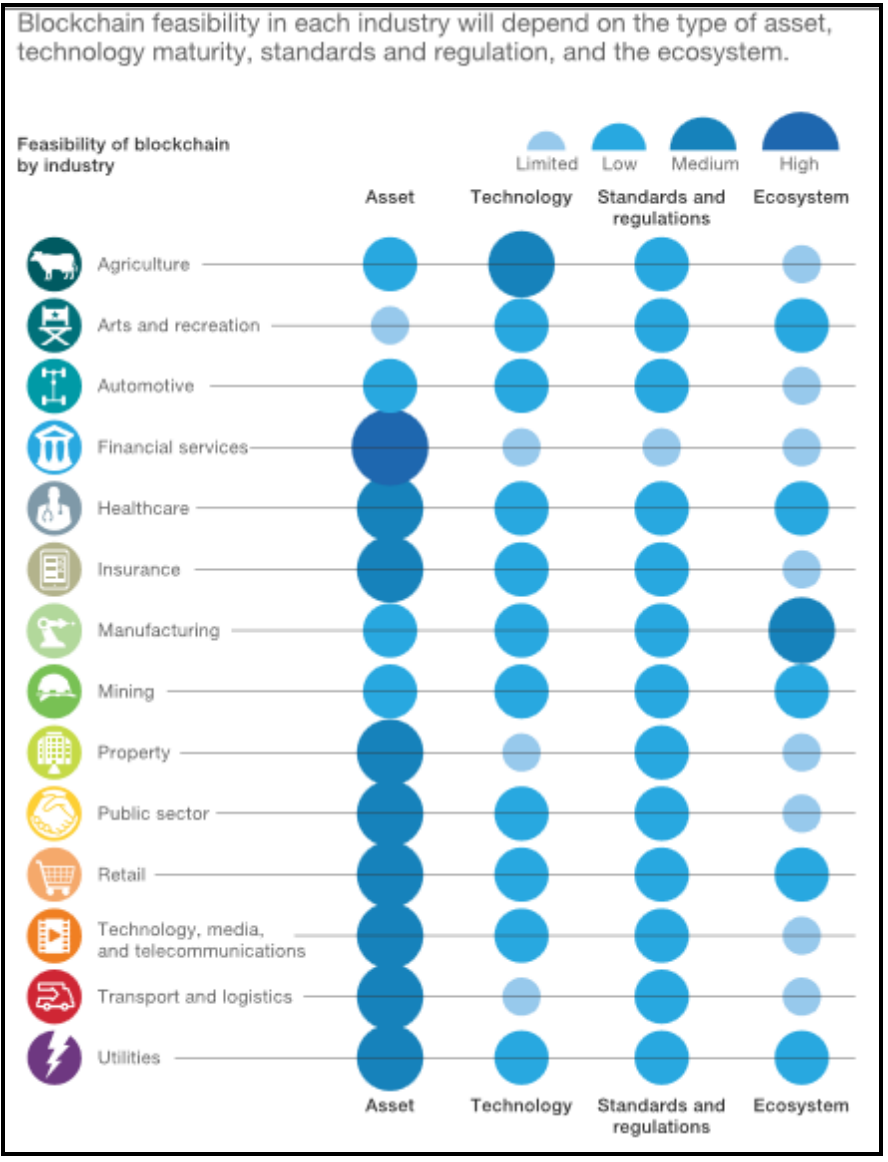

Figure 8: Fintech, Mckinsey\&Company, Feasibility

\section{INDUSTRY EXPERT TALKS}

This section provides feedback from leading research organizations for Blockchain and how they will be represented in the near future.

Gartner says- Current blockchain technologies and concepts are immature, poorly understood and unproven in mission-critical, at-scale business operations. This is particularly so with the complex elements that support more sophisticated scenarios. "Despite the challenges, the significant potential for disruption means CIOs and IT leaders should begin evaluating blockchain, even if they don't aggressively adopt the technologies in the next few years."

Among the 600 executives surveyed by PwC, 25 percent claim to have fully live blockchains implementations or pilot programs already underway. Furthermore, 32 percent of executives say their companies have blockchain implementations in active development, while 20 percent are in a research phase for determining how to best use blockchain technology. In total, only 14 percent of surveyed executives stated that there was no interest in blockchain technology at their companies. Despite widespread interest in using blockchain technology, executives see potential hurdles. Forty-eight percent listed regulatory uncertainty among the top three barriers to blockchain adoption, and 27 percent rating it as the most pressing issue. A lack of trust among users was rated a close second, with 45 percent of respondents placing it in the top three and 25 percent ranking it as the top issue. Technical considerations followed; 44 percent of respondents were concerned about their ability to bring their network together, and 41 percent were concerned about separate blockchains not working together. Rounding out the top seven concerns were questions about blockchain's ability to scale, intellectual property concerns, and audit or compliance concerns. [6]

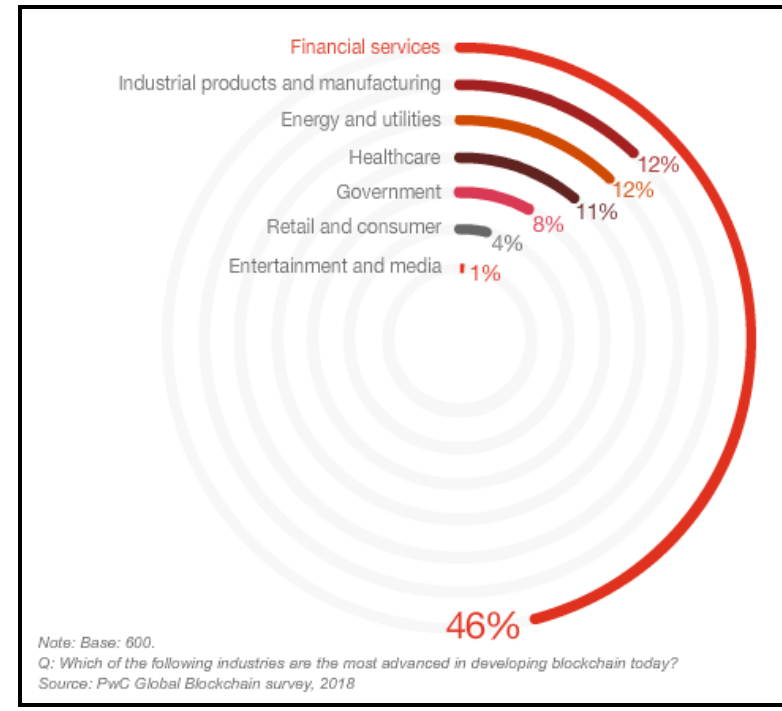

Figure 9: Fintech, Barriers to Enterprise Blockchain Adoption

\section{CONCLUSIONS}

Given the horizon for complete and inspired blockchain solutions, be careful about the assumptions you have going into various use cases. Seriously consider potential use cases and how blockchain fits into your organization's risk profile and investment scenarios. Many people are still trying to understand what blockchain really is and what it could offer.

Interest in blockchain continues unabated among enterprises. Much of the focus is educational and experimental as executives struggle to understand concepts and applicability. Industries have made some progress, but maturity timelines are uncertain, and adoption at scale remains very scarce.

Scalability concerns will continue to hinder enterprise adoption of blockchain. Enterprise architecture and technology innovation leaders must assess technical and operating model scalability factors that will lead to global blockchain scalability by 2022 and plan their projects accordingly.

The value will take time to accrue. Nothing is really happening for several years. Be careful about promising anything to the business and show them the reality of the current situation.

Gartner research concludes with these main strategic planning assumptions: [6]

1. By 2023, viable permissioned blockchains will be tightly integrated with public blockchains, using architectural options such as sidechains hanging off, of public blockchains.

2. By 2020, most permissioned blockchains will be anchored to public blockchains using one of various technical methods, such as sidechains or virtual chains, but their scalability and operational effectiveness will not be widely proven until 2022 
3. By 2022, the dominant blockchain platforms will support the flow of both private and public transactions across multiple ledgers.

4. By 2028, public and permissioned blockchains will be merged at the infrastructure level, and they will support transactions that are either public or private.

\section{CITATIONS}

[1] IJERT, "Blockchain for Supply Chain and Manufacturing Industries and Future It Holds!", Retrieved from, IJERTV7IS090020, http://dx.doi.org/10.17577/IJERTV7IS090020

[2] Gartner Press Release, (May 03, 2018), "Gartner Survey Reveals the Scarcity of Current Blockchain Deployments", Retrieved from, https://www.gartner.com/newsroom/id/3873790

[3] Jill Beadle, (Oct 18, 2018), "Digital Disruption Myths", Retrieved from, https://www.gartner.com/smarterwithgartner/digital-disruptionmyths/?utm_campaign=RM_NA_2018_SWG_NL_NL43_PI\&utm_me
dium=email\&utm_source=Eloqua\&cm mmc=Eloqua-_-Email-_LM_RM_NA_2018_SWG_NL_NL43_PI-_-0000

[4] Heather Pemberton Levy, (Oct 16, 2018), "The Reality of Blockchain", Retrieved From, https://www.gartner.com/smarterwithgartner/thereality-of-

blockchain/?utm_campaign=RM_NA_2018_SWG_NL_NL43_PI\&utm _medium=email\&utm_source=Eloqua\&cm_mmc=Eloqua-_-Email-_LM_RM_NA_2018_SWG_NL_NL43_PI- -0000

[5] Brant Carson, Giulio Romanelli, Patricia Walsh, and Askhat Zhumaev, McKinsey \& Company, (June, 2018), "Blockchain beyond the hype: What is the strategic business value?", Retrieved From, https://www.mckinsey.com/business-functions/digital-mckinsey/ourinsights/blockchain-beyond-the-hype-what-is-the-strategic-businessvalue

[6] Avivah Litan, (Sep 24, 2018), "The Shortsightedness of Blockchain Disillusionment", Retrieved from, https://blogs.gartner.com/avivahlitan/2018/09/24/the-shortsightedness-of-blockchain-disillusionment/ 